\title{
Tratamiento con radiofrecuencia de la tormenta eléctrica: evolución y seguimiento
}

\author{
Radiofrequency treatment for electrical storm: evolution and monitoring
}

\author{
Pablo M. Ruiz-Hernández ${ }^{1,2,3}$, Felipe Atienza1,3*, Leonel Díaz-González,3, Gerard Loughlin ${ }^{1,3}$, \\ Esteban G. Torrecilla1,2,3, Pablo Ávila ${ }^{1,3}$, Tomás Datino ${ }^{1,3}$, Ángel Arenal ${ }^{1,3} y$ \\ Francisco Fernández-Avilés ${ }^{1,2,3}$
}

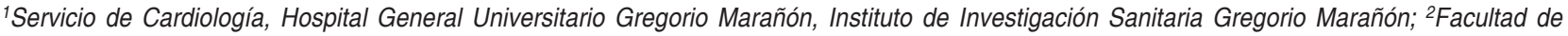
Medicina, Universidad Complutense de Madrid, ${ }^{3}$ Centro de Investigación Biomédica en Red de Enfermedades Cardiovasculares (CIBERCV), Instituto de Salud Carlos III. Madrid, España

\begin{abstract}
Resumen
Introducción y objetivos: La tormenta eléctrica (TE) se caracteriza por episodios repetidos de taquicardia ventricular o fibrilación ventricular relacionados con mal pronóstico a corto y largo plazos. El objetivo fue evaluar la prevalencia, resultados y supervivencia de los pacientes sometidos a tratamiento intervencionista por TE en un centro de referencia. Métodos: Estudio unicéntrico, observacional y retrospectivo. Se revisaron los procedimientos de ablación por TE y se evaluaron las características basales de los pacientes, tipo de procedimiento, mortalidad total, recurrencia de arritmia, mortalidad cardiovascular y necesidad de trasplante. Resultados: Desde enero de 2009 hasta diciembre de 2016 se realizaron 67 procedimientos (38\% de complejos: $19 \%$ de ablación endoepicárdica, $7.5 \%$ de crioablación epicárdica quirúrgica, 3\% de simpatectomía, 3\% de inyección coronaria con alcohol; $6 \%$ de apoyo con oxigenación con membrana extracorpórea) en 41 pacientes (61\% de causa isquémica) por TE. La mortalidad intraprocedimiento fue del 1.5\%. La mediana de seguimiento fue de 23.5 meses (RIQ, 14.2-52.7). Tras el primer ingreso por TE (uno o varios procedimientos), la mortalidad a un año fue de $9.8 \%$. La incidencia acumulada de trasplante cardiaco por TE fue de 2.4\%. En el análisis multivariado, el riesgo de recurrencias arrítmicas o muerte por cualquier causa fue significativamente mayor en pacientes con arritmias clínicas inducibles (HR, 9.03; $p=0.017$ ). Conclusiones: El tratamiento de pacientes con TE, instituido en un centro de referencia y con experiencia, se relacionó con una tasa baja de recurrencia y supervivencia elevada, con una tasa de trasplante cardiaco por TE muy baja. Ante una recurrencia temprana es recomendable practicar un nuevo procedimiento durante el ingreso.
\end{abstract}

Palabras clave: Tormenta eléctrica. Ablación con catéter. Taquicardia ventricular. Fibrilación ventricular.

\begin{abstract}
Introduction and objective: Electrical storm (ES) is characterized by repeated episodes of ventricular tachycardia or ventricular fibrillation, with poor short and long term prognosis. Our objective was to evaluate the prevalence, results of
\end{abstract}

Correspondencia:

*Felipe Atienza

E-mail: fatienzaf@secardiologia.es

DOI: $10.24875 / A C M .20000345$
Disponible en internet: $23-12-2020$ Arch Cardiol Mex. 2020;90(4):379-388 www.archivoscardiologia.com 1405-9940 / (c) 2020 Instituto Nacional de Cardiología Ignacio Chávez. Publicado por Permanyer. Este es un artículo open access bajo la licencia CC BY-NC-ND (http://creativecommons.org/licenses/by-nc-nd/4.0/). 
interventional treatment and survival of patients undergoing interventional treatment for ES in our center. Methods: Retrospective, unicentric and observational study. ES ablation procedures were revised and data regarding baseline characteristics of the patients, type of procedure, total mortality, recurrence of arrhythmia, cardiovascular mortality and the need for transplantation were evaluated. Results: From January 2009 to December 2016, 67 procedures (38\% complex procedures: 19\% epicardial ablation, $7.5 \%$ surgical epicardial crioablation, 3\% simpatectomy, 3\% coronary alcohol injection, $6 \%$ extracorporeal membrane oxygenation support) were performed in 41 patients (61\% Ischemic etiology) due to ES. Intraprocedural mortality was 1.5\%. The median follow-up was 23.5 months (IQR [14.2-52.7]). After the first admission for ES (one or several procedures), 1-year mortality was $9.8 \%$. The cumulative incidence of cardiac transplantation was $2.4 \%$. The risk of arrhythmic recurrences or death was significantly higher in patients with inducible clinical arrhythmias after ablation (HR: 9.03, $p=0.017$ ). Conclusions: The treatment of patients with ES, performed in a reference center, allows obtaining good rates of recurrence and survival, with very low rates of cardiac transplantation for ES. In the presence of an early recurrence, it is advisable to perform a new procedure during admission.

Key words: Electrical storm. Catheter ablation. Ventricular tachycardia. Ventricular fibrillation.

\section{Introducción}

La tormenta eléctrica (TE) es un cuadro clínico caracterizado por episodios repetidos de taquicardia ventricular (TV) o fibrilación ventricular (FV) que requiere tres o más intervenciones en 24 horas para su terminación ${ }^{1}$. Se observa en un $10 \%$ de los pacientes evaluados por descargas del desfibrilador automático implantable $(\mathrm{DAl})^{2}$ y hasta en $4 \%$ de los pacientes con DAI en prevención primaria de origen isquémico ${ }^{3}$. El pronóstico es muy adverso a corto y largo plazo, con evidencia de aumento de mortalidad, trasplante cardiaco y hospitalización, además de acompañarse de una tasa elevada de recurrencia de arritmias ventriculares $^{4-6}$. En pacientes con TE resistente al tratamiento médico óptimo y apoyo hemodinámico, la ablación con catéter por radiofrecuencia es el tratamiento de elección ${ }^{7-12}$ y se considera una emergencia electrofisiológi$\mathrm{ca}^{4,8}$. Las guías de práctica clínica recomiendan remitir a estos pacientes a centros especializados de forma temprana para su atención $n^{7,9,10}$.

La experiencia publicada hasta la fecha es muy escasa, centrada en la ablación con radiofrecuencia, y ninguna serie ha evaluado el tratamiento intervencionista integral de la $T E^{11,13}$. El objetivo de este estudio fue evaluar la prevalencia, los resultados del tratamiento invasivo multimodal y los resultados a corto y largo plazos de los pacientes con TE resistente al tratamiento farmacológico en un centro de referencia.

\section{Métodos}

\section{Pacientes}

Se revisó a todos los pacientes sometidos al menos a un procedimiento terapéutico de ablación por TE en el centro de los autores, entre el 1 de enero del 2009 y el 31 de diciembre de 2016. Todos los enfermos ingresaron en la Unidad de Cuidados Intensivos Cardiológicos, donde se efectuaron el control de los factores precipitantes, la vigilancia continua, la reprogramación del DAI y el tratamiento hemodinámico avanzado, incluidos el balón de contrapulsación o la implantación del sistema de asistencia mediante oxigenación por membrana extracorpórea (ECMO). Los pacientes recibieron tratamiento mediante sedación, administración de antiarrítmicos, bloqueo simpático farmacológico o intubación orotraqueal. Ante la falta de respuesta al tratamiento médico se solicitaron un estudio electrofisiológico u otras medidas invasivas. Sólo se incluyó en este estudio a los pacientes que fueron objeto de al menos un procedimiento terapéutico invasivo dirigido a eliminar la arritmia. El estudio tuvo aprobación del Comité Ético de Investigación con medicamentos del propio centro.

\section{Procedimientos de intervencionismo cardiovascular}

Se revisaron todos los procedimientos terapéuticos realizados en pacientes por una TE. Estos procedimientos incluyeron estudios electrofisiológicos de ablación con catéter por radiofrecuencia (vía endocárdica, epicárdica o mixta), crioablación epicárdica mediante abordaje quirúrgico, simpatectomía, ablación con inyección de alcohol en una rama coronaria o trasplante cardíaco. Todos los procedimientos de ablación con catéter por radiofrecuencia se llevaron a cabo mediante catéter de punta irrigada y sistemas de navegación electroanatómica $\mathrm{CARTO}^{\circledR}$ o EnSite-NavX ${ }^{\circledR}$; el acceso (endocárdico/epicárdico) y la forma de ablación (abordaje de TV clínicas: técnica común ${ }^{12} 0$ ablación de sustrato ${ }^{13}$ ) los eligió el operador según su criterio y 
las características del paciente. La decisión para realizar el procedimiento con asistencia ventricular mediante ECMO se tomó tras considerar la situación clínica del enfermo y de acuerdo con el equipo de la Unidad de Cuidados Intensivos Cardiológicos.

El seguimiento de los pacientes se efectuó al primer, tercer y sexto meses y a continuación con seguimiento semestral. Se revisaron las historias clínicas y en los individuos procedentes de otros centros se recabó la información necesaria mediante contacto personal con los pacientes o médicos de referencia.

\section{Variables de estudio}

Como variable principal se consideró la combinación de la recurrencia de la arritmia ventricular o la muerte por cualquier causa. Como variables secundarias se analizaron la recurrencia de TE, recurrencia de arritmia ventricular (TV/FV), la muerte por cualquier causa, la muerte cardiovascular y la necesidad de trasplante por TE. Se analizó la eficacia del procedimiento a largo plazo según los resultados de la prueba de inducibilidad de TV/FV posterior a la ablación: ausencia de arritmias inducibles (grupo A), inducción de arritmias no clínicas (grupo B), inducción de arritmias clínicas (grupo C), y falta de realización de la prueba de inducibilidad posterior a ablación (grupo D).

\section{Análisis estadístico}

Las variables categóricas se expresaron como número y porcentaje, y las variables continuas como media \pm desviación estándar. Para las comparaciones entre variables categóricas se emplearon las pruebas de la П cuadrada o la de Fisher. La comparación entre variables cuantitativas se llevó a cabo mediante $t$ de Student. El análisis de la supervivencia, la supervivencia libre de recurrencia y su combinada se realizó mediante la prueba de Kaplan-Meier. Se empleó la prueba log-rank para las comparaciones de supervivencia. Para el análisis multivariado de riesgo de episodios adversos se recurrió a la prueba de Cox. El análisis estadístico se efectuó con SPSS 18.0 y programa R, versión 3.3.1. Se consideró significativo un valor de $\mathrm{p}<0.05$.

\section{Resultados}

Entre enero de 2009 y diciembre de 2016 se llevó a cabo en este centro un total de 67 procedimientos terapéuticos por TE en 41 pacientes (1.63 procedimientos/
Tabla 1. Características basales

\begin{tabular}{|c|c|}
\hline & $n=41$ \\
\hline Sexo masculino $\mathrm{n}(\%)$ & $36(87.8)$ \\
\hline NYHA previa, $\mathrm{n}(\%)$ & $\begin{array}{c}\text { I: } 22(53.6 \%) \\
\text { II: } 15(36.5 \%) \\
\text { III: } 3(7.3 \%) \\
\text { IV: } 0(0 \%) \\
\text { Sin datos: } 1\end{array}$ \\
\hline Edad (media \pm DT) & $61.1 \pm 16.2$ \\
\hline Causas, n (\%) & $\begin{array}{c}\text { Isquémica } 25(61) \\
\text { No isquémica } 15(36.6) \\
\text { MCA } 3(7.3) \\
\text { MCH } 1(2.4) \\
\text { MCD } 11(26.8) \\
\text { Sin cardiopatía estructural } 1 \\
\text { (2.4) }\end{array}$ \\
\hline FEVI (mediana, IQR) & $36(20-45)$ \\
\hline $\begin{array}{l}\text { FEVI conservada }(\geq 50 \%) \text {, } \\
\text { n }(\%)\end{array}$ & $3(7.3)$ \\
\hline $\begin{array}{l}\text { Pacientes con } \\
\text { procedimientos de ablación } \\
\text { de TV previos, } \mathrm{n}(\%)\end{array}$ & $19(46.3)$ \\
\hline
\end{tabular}

NYHA: clase funcional de la New York Heart Association; FEVI: fracción de expulsión del ventrículo izquierdo; IQR: intervalo intercuartílico; TV: taquicardia ventricular; MCA: miocardiopatía arritmogénica; $\mathrm{MCH}$ : miocardiopatía hipertrófica; MCD: miocardiopatía dilatada.

paciente). Treinta y seis casos (87.8\%) correspondieron a hombres, con una edad media de $61.1 \pm 16.2$ años y 25 individuos (61\%) presentaban causas isquémicas. La FEVI media fue del $34.2 \pm 13 \%$, con 3 pacientes $(7.3 \%)$ con $\mathrm{FEVI}>50 \%$ y $22(53.6 \%)$ sin limitación previa de la clase funcional (media CF-NYHA: $1.49 \pm 0.7$ ). Hasta 19 sujetos (46.3\%) se habían sometido al menos a un procedimiento de ablación con radiofrecuencia antes del primer procedimiento por TE (Tabla 1). Se excluyó a los pacientes con intoxicación farmacológica, alteraciones electrolíticas graves 0 isquemia aguda no resuelta. En 23 pacientes (56.1\%) se efectuó una coronariografía en las 48 horas anteriores a su primer procedimiento terapéutico.

Se practicaron 67 intervenciones terapéuticas, de las cuales $45(62.2 \%)$ fueron ablaciones endocárdicas, $6(9 \%)$ epicárdicas, 7 (10.4\%) procedimientos de ablación endocárdica-epicárdica, 5 (7.5\%) crioablación epicárdica con acceso quirúrgico, 2 (3\%) simpatectomías y $2(3 \%)$ ablaciones mediante inyección coronaria de alcohol. La tabla 2 muestra la eficacia aguda de los distintos procedimientos y las recurrencias de TE a largo plazo tras estos procedimientos. 
Tabla 2. Tipos de procedimientos

\begin{tabular}{|l|c|c|c|}
\hline & Procedimientos & $\begin{array}{c}\text { Éxito } \\
\text { agudo* }\end{array}$ & $\begin{array}{c}\text { Éxito } \\
\text { crónico** }\end{array}$ \\
\hline $\begin{array}{l}\text { Ablación } \\
\text { endocárdica }\end{array}$ & 45 & $34(75.5 \%)$ & $22(48.9 \%)$ \\
\hline $\begin{array}{l}\text { Ablación } \\
\text { epicárdica }\end{array}$ & 6 & $6(100 \%)$ & $2(33.3 \%)$ \\
\hline $\begin{array}{l}\text { Ablación } \\
\text { endocárdica- } \\
\text { epicárdica }\end{array}$ & 7 & $6(85.7 \%)$ & $5(71.4 \%)$ \\
\hline \begin{tabular}{l} 
Simpatectomía \\
\hline Alcohol
\end{tabular} & 2 & $1(50 \%)$ & $1(50 \%)$ \\
\hline $\begin{array}{l}\text { Crioablación } \\
\text { quirúrgica }\end{array}$ & 5 & $1(50 \%)$ & $1(50 \%)$ \\
\hline Total & 67 & $52(77.6 \%)$ & $33(49.2 \%)$ \\
\hline
\end{tabular}

*Ausencia de arritmia clínica al finalizar el procedimiento

**Ausencia de arritmia clínica durante el seguimiento.

Al llegar al Laboratorio de Electrofisiología se identificó la presencia de arritmia clínica espontánea en $18(43.9 \%)$ de los procedimientos (Tabla 3). No hubo diferencias significativas en la eficacia aguda del procedimiento en pacientes con arritmia clínica espontánea (14/18 pacientes, $77.8 \%$ ) y aquéllos sin arritmia durante la ablación (20/23 pacientes, $87 \%)$. Se empleó una ablación común en $13(20.6 \%)$, sin incluir cuatro procedimientos de simpatectomía y ablación con alcohol, y una ablación de sustrato en 50 (79.4\%). En el primer caso, el ciclo medio de la taquicardia clínica espontánea o inducida fue de $370.2 \pm 60.3 \mathrm{~ms}$ (mediana, $360 \mathrm{~ms} ;$ IQR, 320-410). Un paciente falleció en 2014 durante el estudio electrofisiológico por hipotensión progresiva y choque resistente (1/67, primer y único procedimiento practicado en ese paciente; Tabla 1, material complementario). En el año 2015 se inició la implantación de un programa de ECMO para la ablación de TE en este centro y se efectuaron cuatro procedimientos (4/23, $17.4 \%$ en esos años) con apoyo de ECMO intraprocedimiento, tres durante la ablación endocárdica y uno endocárdico-epicárdico. En estos cuatro casos, el sistema ECMO permitió llevar a cabo el procedimiento con adecuada estabilidad hemodinámica. Las simpatectomías $(n=2)$ se efectuaron mediante punción percutánea y aplicación de anestésico sobre el ganglio estrellado, con una eficacia aguda del $50 \%$. Los procedimientos de ablación con alcohol $(n=2)$ se realizaron en pacientes con miocardiopatía no isquémica, con arritmia ventricular de origen septal a nivel
Tabla 3. Características del procedimiento

\begin{tabular}{|c|c|}
\hline $\begin{array}{l}\text { Procedimientos en TE por } \\
\text { paciente }\end{array}$ & $\begin{array}{c}1=28(68.3) \\
2=6(14.6) \\
3=4(9.7) \\
4=1(2.4) \\
5=1(2.4) \\
6=1(2.4)\end{array}$ \\
\hline $\begin{array}{l}\text { Coronariografía } 48 \mathrm{~h} \\
\text { previas }\end{array}$ & $28(42.8 \%)$ \\
\hline $\begin{array}{l}\text { Arritmia clínica espontánea } \\
\text { al inicio del primer } \\
\text { procedimiento }\end{array}$ & $18(43.9 \%)$ \\
\hline $\begin{array}{l}\text { Ciclo TV (ms) en el primer } \\
\text { procedimiento } \\
\text {-(media, DT) } \\
\text {-(mediana; IQR; intervalo) }\end{array}$ & $\begin{array}{c}370.2 \pm 60.3 \mathrm{~ms} \\
360 \mathrm{~ms} ; 320-410 ; 280-500\end{array}$ \\
\hline $\begin{array}{l}\text { Procedimientos } \\
\text { endocárdicos por paciente }\end{array}$ & $\begin{aligned} 1 & =25(61) \\
2 & =10(24.4) \\
3 & =1(2.4) \\
4 & =1(2.4)\end{aligned}$ \\
\hline $\begin{array}{l}\text { Procedimientos } \\
\text { epicárdicos por paciente }\end{array}$ & $\begin{array}{l}1=11(26.8) \\
2=1(2.4)\end{array}$ \\
\hline $\begin{array}{l}\text { Procedimientos quirúrgicos } \\
\text { por paciente }\end{array}$ & $1=5(12.2)$ \\
\hline $\begin{array}{l}\text { Procedimientos por } \\
\text { extrasístoles ventriculares } \\
\text { que induce FV por paciente }\end{array}$ & $\begin{array}{l}1=4(9.7) \\
2=2(4.9)\end{array}$ \\
\hline Ventrículo tratado & $\begin{array}{l}\text { Izquierdo: } 60 \text { (89.5) } \\
\text { Derecho: } 6 \text { (8.9) } \\
\text { Ambos: } 1 \text { (1.5) }\end{array}$ \\
\hline $\begin{array}{l}\text { Ablación } \\
\text {-Común } \\
\text {-Sustrato }\end{array}$ & $\begin{array}{l}13(20.6)^{*} \\
50(79.4)^{*}\end{array}$ \\
\hline ECMP & $4(6)$ \\
\hline $\begin{array}{l}\text { Éxito agudo del primer } \\
\text { procedimiento }\end{array}$ & $34(82.9)$ \\
\hline Mortalidad & $1(1.5)$ \\
\hline Complicaciones & 1 Ictus (1.5) \\
\hline $\begin{array}{l}\text { Inducibilidad al final del } \\
\text { estudio**, } \mathrm{n}(\%)\end{array}$ & $\begin{array}{c}\text { Grupo A (ninguna): } 22(37.3 \%) \\
\text { Grupo B (no clínicas): } 9(15.2 \%) \\
\text { Grupo C (clínicas): } 6(10.2 \%) \\
\text { Grupo D (no evaluado): } 22(37.3 \%)\end{array}$ \\
\hline
\end{tabular}

Número (\%); media $\pm \mathrm{DE}$.

*Excluidos en el porcentaje de cuatro procedimientos (dos simpatectomías y dos ablaciones con alcohol).

${ }^{* *}$ Se excluye FV inducida por extrasístoles.

de la porción más superior del ventrículo izquierdo (summit) y tabique interventricular alto, en pacientes con intento previo ineficaz de ablación por radiofrecuencia, y con una rama coronaria apropiada para su oclusión, con una eficacia aguda del 50\%. De los 41 


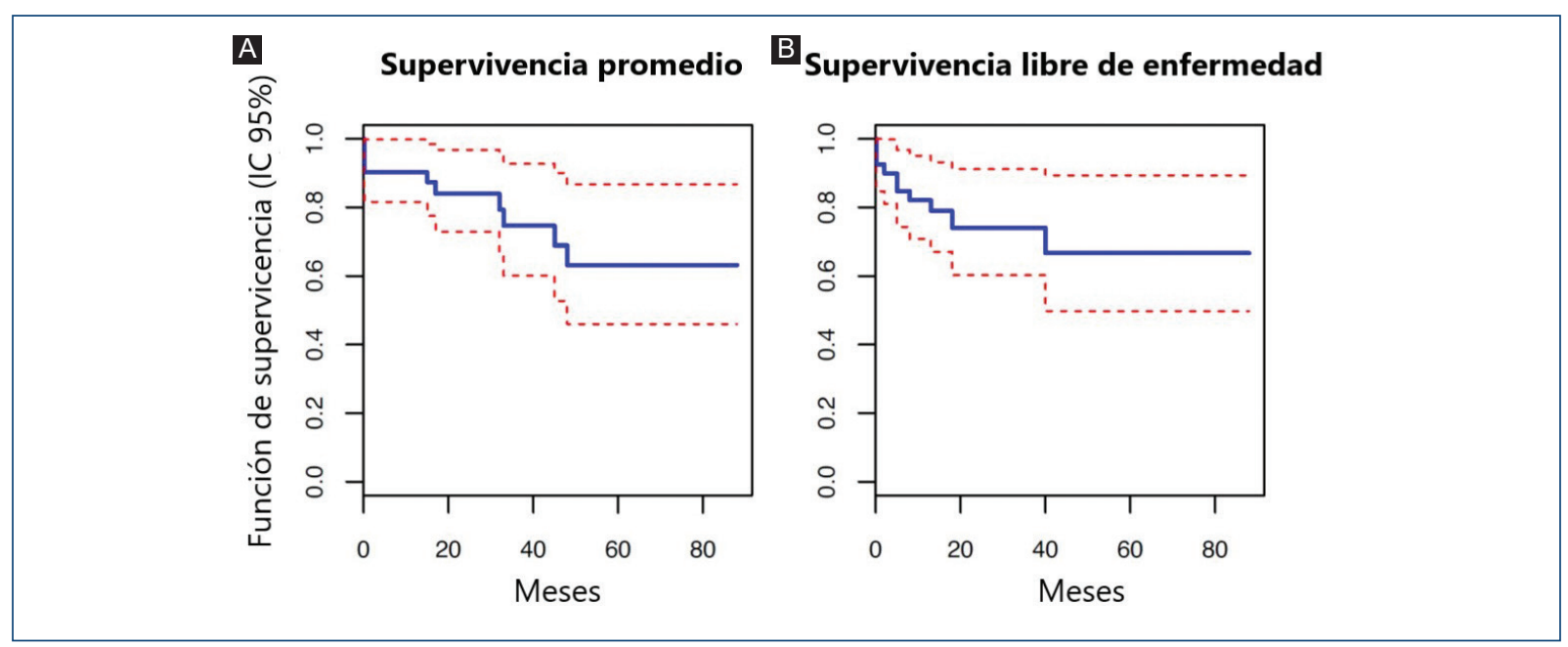

Figura 1. A: Curva de supervivencia. B: Supervivencia libre de recurrencia tras primer ingreso por tormenta eléctrica.

pacientes, 14 (34.1\%) presentaron recurrencia de arritmia ventricular tras el primer procedimiento durante el ingreso y, luego de los tratamientos adicionales, $57 \%$ de ellos (8 pacientes) no mostró recurrencias después del primer ingreso (Fig. 1B).

Durante el período estudiado (8 años) se completaron 1,290 meses de seguimiento en el total de pacientes (mediana: 24 meses; RIQ, 14.5-52.5). El tiempo medio de supervivencia tras el primer ingreso por TE fue de $65 \pm 6.1$ meses (IC95\%, 53.1-76.8; Fig. 1A). Luego del primer ingreso por TE (uno o varios procedimientos), la mortalidad a un año fue del $9.8 \%$. La mortalidad al final del seguimiento fue del $24.4 \%$ (10/41): 6 pacientes (60\%) por muerte cardiovascular, 4 de ellos durante el ingreso: un caso de choque cardiogénico durante el procedimiento de ablación, dos recurrencias de TE durante la primera semana después del procedimiento en pacientes no elegibles para trasplante cardíaco (por edad y comorbilidad) con reintervenciones fallidas, y un paciente por sepsis diferida previamente sometido a ablación bajo asistencia de ECMO (Tabla 1, material complementario). Los casos de mortalidad cardiovascular tardía $(n=2)$ se debieron a insuficiencia cardíaca terminal. Cuatro sujetos fallecieron por causa no cardíaca: uno por choque séptico, uno por neumonía complicada, uno por choque distributivo en un contexto de colitis isquémica, y uno por muerte no súbita por causa desconocida. Durante el seguimiento, un individuo requirió trasplante cardíaco por recurrencia de TE sin respuesta al tratamiento (2.4\%). Se evaluó la inducibilidad de TV/FV al final de la primera intervención en 25 pacientes (61\%): 2 (8\%) presentaron arritmias clínicas (grupo $\mathrm{C}$, sin éxito), $6(24 \%)$ arritmias no clínicas (grupo B, éxito parcial) y $17(68 \%)$ no experimentaron arritmias inducibles (grupo A, éxito completo); en 16 sujetos no se evaluó la inducibilidad al final del primer procedimiento por TE, sin incluir a aquéllos con FV inducida por extrasístoles o mala situación clínica (grupo D). En el análisis multivariado, sólo la inducibilidad de las arritmias clínicas fue predictor de muerte o recurrencia de arritmias (HR, 9.034; IC95\%, 1.49-54.78; $p=0.017$ ), luego del primer procedimiento terapéutico por TE (Tabla 4). No se observaron diferencias significativas en cuanto al riesgo de muerte o recurrencia de TV/FV, en función de la clase funcional anterior al episodio (log-rank $=0.461$ ) o la clase funcional de la NYHA. Después del primer ingreso, los pacientes con una causa isquémica, en comparación con la no isquémica, mostraron una tendencia no significativa a una mayor mortalidad por cualquier causa (52 meses [IC95\%, 36.9-67.2 vs. 77.5 meses; IC95\%, 64-90.9; $p=0.08$; Fig. 1, material suplementario). Análisis multivariado: $H R=16.34$, $p=0.055$, tabla 4 .

La figura 2A muestra la supervivencia libre de recurrencia de TV/FV en el seguimiento (grupo $A$ [éxito completo]: $80 \pm 7.3$ meses; grupo B [éxito parcial]: 54.8 \pm 15.1 meses; grupo $C$ [sin éxito]: $4 \pm 3.9$ meses; grupo D [sin prueba]: $46.6 \pm 9.5$ meses). La supervivencia libre de recurrencia de TV/FV en el grupo $C$ fue significativamente menor que en los demás grupos en conjunto ( $p=0.003)$, y en comparación con los grupos $A(p=0.001)$ y $D(p=0.01)$. El resto de comparaciones intergrupales, incluido el grupo $\mathrm{C}$ contra el grupo $\mathrm{B}$ 
Tabla 4. Predictores de mortalidad o recurrencia de TE tras el primer procedimiento de ablación

\begin{tabular}{|c|c|c|c|c|c|c|c|c|c|}
\hline \multicolumn{10}{|c|}{ a) Análisis univariado } \\
\hline & \multicolumn{3}{|c|}{ Recurrencia o muerte } & \multicolumn{3}{|c|}{ Recurrencia } & \multicolumn{3}{|c|}{ Mortalidad } \\
\hline & RR & IC $[5 \%, 95 \%]$ & $\begin{array}{l}\text { Valor } \\
\text { de p }\end{array}$ & RR & IC $[5 \%, 95 \%]$ & $\begin{array}{l}\text { Valor } \\
\text { de } p\end{array}$ & RR & IC $[5 \%, 95 \%]$ & $\begin{array}{l}\text { Valor } \\
\text { de } p\end{array}$ \\
\hline Sexo masculino & 1.18 & {$[0.26,5.24]$} & 0.826 & 0.643 & {$[0.081,5.095]$} & 0.676 & 0.641 & {$[0.081,5.07]$} & 0.673 \\
\hline Edad (por año) & 1.009 & {$[0.976,1.042]$} & 0.601 & 0.989 & {$[0.954,1.026]$} & 0.562 & 1.047 & {$[0.992,1.104]$} & 0.093 \\
\hline $\begin{array}{l}\text { Clase funcional } \geq \text { III } \\
\text { vs. } \leq \mathrm{II}\end{array}$ & 1.722 & {$[0.379,7.827]$} & 0.482 & 1.26 & {$[0.157,10.12]$} & 0.828 & 1.211 & {$[0.153,9.605]$} & 0.856 \\
\hline $\begin{array}{l}\text { Causa isquémica vs. } \\
\text { no isquémica }\end{array}$ & 0.819 & {$[0.289,2.319]$} & 0.707 & 0.522 & {$[0.146,1.864]$} & 0.316 & 3.611 & {$[0.756,17.258]$} & 0.108 \\
\hline FEVI $(\%)$ & 1,012 & {$[0.974,1.152]$} & 0.533 & 1,021 & {$[0.974,1.071]$} & 0.386 & 0.977 & {$[0.931,1.026]$} & 0.358 \\
\hline Ciclo TV (ms) & 1,003 & {$[0.994,1.012]$} & 0.519 & 1,005 & {$[0.994,1.016]$} & 0.38 & 1,003 & {$[0.991,1.015]$} & 0.596 \\
\hline $\begin{array}{l}\text { Arritmia espontánea } \\
\text { EEF \#1 }\end{array}$ & 0.603 & {$[0.24,1.52]$} & 0.284 & 0.485 & {$[0.125,1.877]$} & 0.297 & 0.585 & {$[0.165,2.077]$} & 0.407 \\
\hline EV que genera $\mathrm{FV}^{*}$ & 0.812 & {$[0.182,3.62]$} & 0.785 & 1.175 & {$[0.148,9.315]$} & 0.878 & 0.426 & {$[0.088,2.058]$} & 0.288 \\
\hline $\begin{array}{l}\text { Abl._sustrato vs. } \\
\text { común }\end{array}$ & 2.752 & {$[0.616,12.298]$} & 0.185 & 1.589 & {$[0.333,7.577]$} & 0.561 & 3.654 & {$[0.459,29.111]$} & 0.221 \\
\hline $\begin{array}{l}\text { Inducibilidad de } \\
\text { arritmia clínica }\end{array}$ & 9.034 & {$[1.49,54.783]$} & 0.017 & 14.239 & {$[1.927,102.83]$} & 0.008 & 6.167 & {$[1.107,34.347]$} & 0.038 \\
\hline $\begin{array}{l}\text { Realización de prueba } \\
\text { de inducibilidad }\end{array}$ & 1.117 & {$[0.395,3.156]$} & 0.835 & 1.599 & {$[0.461,5.549]$} & 0.46 & 0.672 & {$[0.173,2.609]$} & 0.566 \\
\hline \multicolumn{10}{|c|}{ b) Análisis multivariado } \\
\hline & \multicolumn{3}{|c|}{ Recurrencia o muerte } & \multicolumn{3}{|c|}{ Recurrencia } & \multicolumn{3}{|c|}{ Mortalidad } \\
\hline & $\mathbf{R R}$ & IC $[5 \%, 95 \%]$ & $\begin{array}{l}\text { Valor } \\
\text { de } p\end{array}$ & $\mathbf{R R}$ & IC $[5 \%, 95 \%]$ & $\begin{array}{l}\text { Valor } \\
\text { de } p\end{array}$ & $\mathbf{R R}$ & IC $[5 \%, 95 \%]$ & $\begin{array}{c}\text { Valor de } \\
\text { p }\end{array}$ \\
\hline $\begin{array}{l}\text { Causa isquémica vs. } \\
\text { no isquémica }\end{array}$ & & & & & & & 16.34 & [0.94 - 283.22] & 0.055 \\
\hline $\begin{array}{l}\text { Inducibilidad Arritmia } \\
\text { clínica }\end{array}$ & 9.03 & {$[1.49-54.78]$} & 0.017 & 14.24 & [1.19 - 102.83] & 0.008 & 28.24 & [2.38 - 333.45] & 0.008 \\
\hline
\end{tabular}

FEVI: fracción de expulsión del ventrículo izquierdo; EEF \#1: primer estudio electrofisiológico por tormenta eléctrica; EV: extrasístole ventricular; FV: fibrilación ventricular. Abl.: ablación.

$(p=0.15), \quad$ no alcanzó significación estadística. La recurrencia de TV/FV (Fig. 2B) en pacientes con arritmias inducibles (grupo $\mathrm{C}$ ) fue significativamente mayor que en el resto de los grupos ( $p<0.05$ para todas las comparaciones) $y$, además, estadísticamente superior en el grupo $D$ respecto del grupo $A(p=0.01)$. Las demás comparaciones intergrupales, incluido el grupo A contra el grupo $B(p=0.061)$, no alcanzaron significación estadística. La mortalidad por cualquier causa (Fig. 2C) fue significativamente mayor en el grupo $\mathrm{C}$ que en el resto de los grupos en conjunto $(p=0.017)$, pero no en comparaciones intergrupales ( $C$ vs. $A$, $p=0.069 ; C$ vs. $B, p=0.118 ; C$ vs. $D=0.058$ ). Las otras comparaciones no mostraron significación estadística. No hubo diferencias entre grupos respecto del tiempo hasta la mortalidad cardiovascular (Fig. 2D, p > 0.05 para todas las comparaciones).

\section{Discusión}

Este estudio muestra que el tratamiento de los pacientes con TE resistente al tratamiento farmacológico habitual, instituido en un centro de referencia y alto volumen, y basado en el uso de la ablación con radiofrecuencia y distintos tratamientos invasivos, permite obtener favorables tasas de episodios en el 


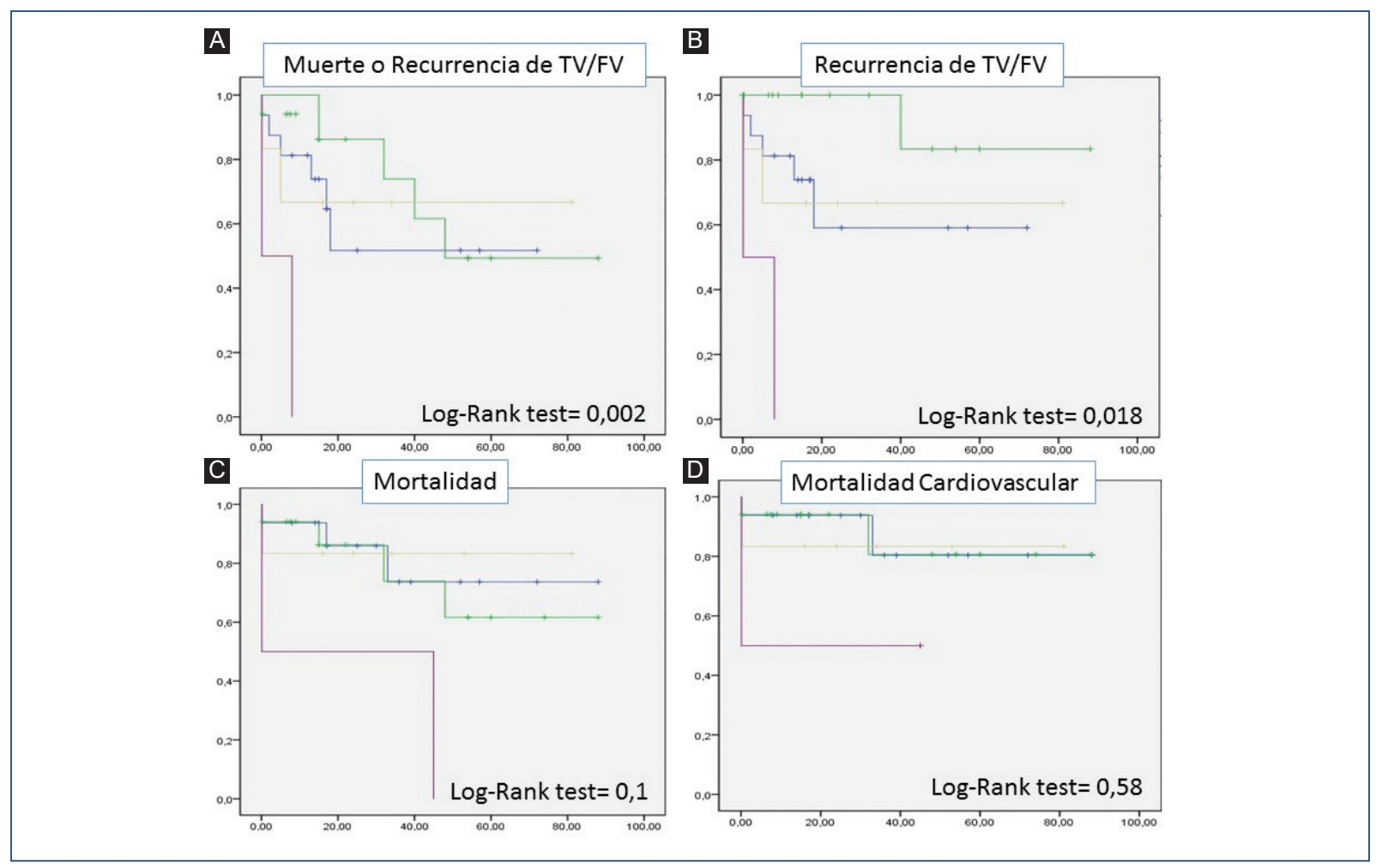

Figura 2. Supervivencia libre de recurrencia o muerte por cualquier causa o cardiovascular, en relación con la inducibilidad de arritmias al final del primer procedimiento. Grupo A (verde): ausencia de arritmias inducibles; grupo B (marrón): inducibilidad de arritmias no clínicas; grupo C (violeta): inducibildiad de arritmias clínicas; grupo D (azul): inducibilidad no evaluada. Se muestra el valor estadístico de log-rank global de inducibilidad para cada episodio evaluado.

seguimiento, en comparación con las publicadas en fecha reciente ${ }^{10,14}$. Este estudio confirma la necesidad de indicar un tratamiento invasivo y global, que de forma escalonada incluya distintos abordajes terapéuticos de la TE y utilice fuentes de energía alternativas, accesos endoepicárdicos o quirúrgicos, así como técnicas antiarrítmicas complejas y especializadas, como la ablación simpática o con alcohol ${ }^{15,16}$. Por último, este estudio apoya la utilidad de los sistemas de asistencia ventricular para la atención de pacientes con TE y deterioro hemodinámico para efectuar el procedimiento con éxito ${ }^{17}$. Todo ello ha permitido mejorar las cifras de supervivencia sin recurrencias arrítmicas dentro de un tratamiento muy especializado, con la participación de distintos profesionales y especialistas.

\section{Pronóstico del tratamiento intervencionista de la TE}

La tormenta eléctrica es una emergencia electrofisiológica vital, definida por la presencia de tres o más episodios de TV o FV en 24 horas, representa 10 a $30 \%$ de los casos de ablación de TV/FV y se relaciona con un incremento significativo de la mortalidad ${ }^{1-6}$. La ablación con catéter reduce las descargas por TV/FV y la mortalidad y prolonga la supervivencia ${ }^{18}$.

La TE es una situación clínica extremadamente compleja debido a la interacción de los efectos de la crisis de inestabilidad eléctrica, las descargas múltiples del desfibrilador y la situación de insuficiencia cardíaca avanzada, en la cual la ablación con frecuencia no se realiza o se consideraba una maniobra de rescate como transición a un trasplante cardíaco ${ }^{4,11,19}$. Los primeros estudios publicados mostraron que la ablación de la TE interrumpe la inestabilidad arrítmica en una elevada proporción de casos; empero, debido al limitado tamaño de los estudios y a la gravedad de la situación clínica de los pacientes, no fue posible demostrar una mejora significativa de la supervivencia global en comparación con la TE tratada de forma no invasiva $^{14,20}$. Sin embargo, estudios de grupos con gran volumen y el reciente registro multicéntrico 
internacional de ablación de TV demuestran que la ablación de TE incrementa la supervivencia a largo plazo, casi de $90 \%$ al año en pacientes con ablación eficaz ${ }^{10,14}$. Este estudio registró una supervivencia al año del $90.2 \%$ y una supervivencia global libre de recurrencias de TV/FV tras el primer ingreso del $85 \%$. A pesar de la complejidad de los procedimientos realizados (38\% de ablaciones complejas) y las necesidades de apoyo con ECMO, la mortalidad temprana $(9.7 \%)$ y la incidencia de complicaciones periprocedimiento (2.4\%) mostraron cifras similares a las de los estudios recientes ${ }^{5,20}$. Por otra parte, sólo hubo un paciente con indicación de trasplante cardíaco por TE (1/126 trasplantes cardiacos, $0.79 \%$ ) durante el período analizado. Estos datos sugieren que la práctica de la intervención en centros de alto volumen reduce de manera muy significativa la necesidad de practicar trasplantes cardíacos de forma urgente en pacientes con TE. Estos datos concuerdan con la reciente actualización de los criterios de los pacientes elegibles para trasplante cardíaco urgente que la ONT estableció en fecha reciente ${ }^{21}$.

\section{Predictores de recurrencia de TV y mortalidad a largo plazo}

La persistencia de arritmias clínicas luego del procedimiento terapéutico demostró ser un factor de mal pronóstico significativo en relación con la recurrencia de arritmia y la mortalidad. Los pacientes sin arritmias clínicas inducibles no presentaron una tasa de recurrencias arrítmicas estadísticamente diferentes respecto de aquéllos con arritmias no clínicas inducibles tras el procedimiento. Por el contrario, en el estudio de Carbucicchio, et al., la ausencia de inducibilidad de cualquier arritmia ventricular se vinculó con menor tasa de recurrencias de arritmias ventriculares que la persistencia de inducibilidad de arritmias no clínicas $^{14}$. No obstante, en la serie del estudio multicéntrico internacional de TV, y en consonancia con los resultados de este estudio, sólo la persistencia de arritmias clínicas inducibles o la falta de la prueba de inducibilidad se acompañaron de mayor tasa de recurrencia de arritmias ventriculare ${ }^{10}$. La utilidad de practicar la ablación continuada hasta eliminar la inducibilidad de cualquier tipo de arritmia prolonga la duración del procedimiento e incrementa el tiempo de radiofrecuencia y el número de desfibrilaciones, lo que podría comprometer la seguridad del paciente a corto plazo ${ }^{22-24}$. En este mismo sentido, estos datos sugieren que la ablación común podría suministrar mejores tasas de recurrencia y mortalidad en comparación con la ablación de sustrato. Sin embargo, en el contexto específico de una causa isquémica de TE, se ha publicado un estudio no aleatorizado y multicéntrico que muestra una reducción de la recurrencias de TV/FV (53\% vs. $81 \%$ ) con una ablación de sustrato extensa endocárdica-epicárdica frente a la ablación regular de sustrato ${ }^{25}$, sin encontrar diferencias en la mortalidad global. La ablación de sustrato, que por definición afecta a una mayor cantidad de tejido, podría acentuar el deterioro funcional en pacientes que ya se encuentran en una situación crítica con grave deterior de la función ventricular y afectación clínica. Por todo ello, en este contexto de TE todavía existen dudas acerca de la necesidad de practicar ablaciones muy extensas que requieran la completa eliminación de todo el sustrato arrítmico necesario para mantener cualquier tipo de arritmia ventricular. Por último, ningún otro factor relacionado con la ablación modificó el pronóstico en la incidencia de recurrencias o mortalidad a largo plazo, como la presencia espontánea de la arritmia clínica durante la ablación ${ }^{20,26}$.

\section{Ablación de la TE y resultados inmediatos}

La ablación con radiofrecuencia con apoyo del sistema de navegación fue la técnica de ablación elegida de manera inicial en todos los pacientes. Aunque la taquicardia ventricular monomórfica fue la situación clínica más prevalente, la variabilidad en las morfologías de TV y la tolerancia hemodinámica exigieron la utilización de medidas avanzadas de mapeo y ablación, así como sistemas de apoyo hemodinámico. Un equipo multidisciplinario condujo los estudios e individualizó las decisiones de acuerdo con la situación basal del paciente, sus preferencias, y la opinión de los profesionales del equipo asistencial. La ablación se llevó a cabo de forma secuencial y la práctica de un procedimiento endocárdico, epicárdico, crioablación epicárdica vía quirúrgica, ablación con alcohol o simpatectomía se decidió en función de la situación clínica, los hallazgos de las técnicas de imagen y los resultados del procedimiento inicial ${ }^{15,16,27}$. En un $32 \%$ se consideró necesaria la repetición de procedimientos de ablación y en 33\% se recurrió a aproximaciones terapéuticas no endocárdicas, con buenos resultados (82\% de éxito agudo), luego de considerar que se trataba de pacientes con procedimientos previos fallidos de ablación endocárdica. En consecuencia, estos datos permiten recomendar una vigilancia especializada y estricta después del procedimiento de ablación y 
repetir el procedimiento de ablación, de acuerdo con todas las técnicas disponibles, ante una recurrencia de TE luego del primer procedimiento.

El uso de ECMO en esta serie es todavía limitado, pero creciente. El papel del apoyo circulatorio en procedimientos con arritmias recidivantes mejora la seguridad y la eficacia del procedimiento, lo que hace posible procedimientos de ablación eficaz y segura en enfermos con arritmias hemodinámicamente ${ }^{17} \mathrm{mal}$ toleradas. La disponibilidad de tecnología avanzada y la experiencia ${ }^{15}$ para la atención multidisciplinaria, sobre todo en relación con estas intervenciones complejas, es la clave de una asistencia integral y de excelencia para pacientes con tormenta eléctrica ${ }^{9}$. La experiencia inicial de los autores sustenta la utilización de sistemas de apoyo hemodinámico en pacientes clínicamente inestables, con TE y malas condiciones hemodinámicas, con una baja incidencia de complicaciones agudas $(2.4 \%)^{28}$.

Este estudio tiene algunas limitaciones. Se trata de un protocolo retrospectivo, por lo que algunas variables clínicas y resultados del procedimiento no están disponibles. El tratamiento antiarrítmico quedó a criterio de los médicos tratantes y tal vez influyó en la evolución clínica. Asimismo, en virtud de su diseño, el estudio muestra el avance de la tecnología disponible para el tratamiento invasivo de las arritmias y su evolución en el tiempo. La ablación no se aleatorizó, sino que se eligió a criterio del operador. Los resultados del análisis multivariado con amplios intervalos de confianza están influidos por el limitado tamaño de la muestra. Aunque se trata de una serie de casos de un centro único, está integrado por un grupo de profesionales estable, con los mismos criterios terapéuticos homogéneos, lo que representa una mayor solidez de los resultados obtenidos.

Como conclusión, el tratamiento de pacientes con TE, realizado en un centro de referencia y con experiencia, basado sobre todo en la ablación con radiofrecuencia, permite obtener unas tasas de recurrencia y supervivencia equivalentes a las publicadas en los centros de referencia internacionales en esta situación clínica, con tasas de trasplante cardiaco por TE muy bajas. El objetivo del procedimiento debe ser no inducir la arritmia clínica y, en caso de recurrencia temprana, es recomendable un nuevo procedimiento durante el ingreso.

\section{Agradecimientos}

Los autores agradecen la colaboración de María Padilla, de la Organización Nacional de Trasplante (España) y del profesor Pedro Saavedra, del
Departamento de Matemáticas de la Universidad de Las Palmas de Gran Canaria (España).

\section{Financiamiento}

Este estudio recibió financiamiento parcial de "Convocatoria de ayudas a la contratación de investigadores RIC 2014", CIBERCV, Instituto de Investigación Carlos III. Becas Pl13-01882, Pl13-00903, PI14/00857, PI16/01123, PI17/01059, TEC2013-46067-R, DTS16/0160 and IJCl-2014- 22178); y fondos FEDER.

\section{Conflicto de intereses}

Ninguno de los autores tiene conflicto de intereses relacionado con el contenido del artículo.

\section{Responsabilidades éticas}

Protección de personas y animales. Los autores declaran que los procedimientos seguidos se ajustan a las normas éticas del comité de experimentación humana responsable y a la Asociación Médica Mundial y la Declaración de Helsinki.

Confidencialidad de los datos. Los autores declaran que han seguido los protocolos de su centro de trabajo sobre la publicación de datos de pacientes.

Derecho a la privacidad y consentimiento informado. Los autores declaran que en este artículo no aparecen datos de pacientes.

\section{Bibliografía}

1. Hohnloser SH, Al-Khalidi HR, Pratt CM, Brum JM, Tatla DS, Tchou P, et al. Electrical storm in patients with an implantable defibrillator: Incidence, features, and preventive therapy: Insights from a randomized trial. Eur Heart J. 2006;27(24):3027-3032.

2. Verma A, Kilicaslan F, Marrouche NF, Minor S, Khan M, Wazni O, et al. Prevalence, predictors, and mortality significance of the causative arrhythmia in patients with electrical storm. J Cardiovasc Electrophysiol. 2004;15(11):1265-1270.

3. Sesselberg HW, Moss AJ, McNitt S, Zareba W, Daubert JP, Andrews ML, et al. Ventricular arrhythmia storms in postinfarction patients with implantable defibrillators for primary prevention indications: a MADIT-II substudy. Heart Rhythm 2007;4(11):1395-402.

4. Bänsch D, Böcker D, Brunn J, Weber M, Breithardt G, Block M. Clusters of ventricular tachycardias signify impaired survival in patients with idiopathic dilated cardiomyopathy and implantable cardioverter defibrillators. J Am Coll Cardiol. 2000;36(2):566-73.

5. Santangeli P, Frankel DS, Tung R, Vaseghi M, Sauer WH, Tzou WS, et al. Early mortality after catheter ablation of ventricular tachycardia in patients with structural heart disease. J Am Coll Cardiol. 2017;69(17): 2105-2115.

6. Guerra F, Shkoza M, Scappini L, Flori M, Capucci A. Role of electrical storm as a mortality and morbidity risk factor and its clinical predictors: a meta-analysis. Europace 2014;16(3):347-53.

7. Priori SG, Blomström-Lundqvist C, Mazzanti A, Blom N, Borggrefe M, Camm J, et al. 2015 ESC Guidelines for the management of patients with ventricular arrhythmias and the prevention of sudden cardiac death: The Task Force for the Management of Patients with Ventricular Arrhythmias and the Prevention of Sudden Cardiac Death of the Europe. Eur Heart J. 2015;36(41):2793-867 
8. Hernández-Hernández JM, Datino T, Arenal Á, Núñez-García A, Fernández-Avilés F. Ventricular fibrillation, an emergency in electrophysiology. Rev Esp Cardiol (Engl. ed) 2012;65(5):482-3.

9. Gorenek B, Blomstrom Lundqvist C, Brugada Terradellas J, Camm AJ, Hindricks G, Huber K, et al. Cardiac arrhythmias in acute coronary syndromes: position paper from the joint EHRA, ACCA, and EAPCI task force. Europace 2014;16(11):1655-1673.

10. Vergara $\mathrm{P}$, Tung R, Vaseghi M, Brombin C, Frankel DS, Di Biase L, et al. Successful ventricular tachycardia ablation in patients with electrical storm reduces recurrences and improves survival. Heart Rhythm 2018;15(1):48-55

11. Cabrera AMM, Cordero AB, Rodríguez JA, Carmona JR, de Teresa Galván E. Radiofrequency catheter ablation of ventricular tachycardia in patients with an implantable defibrillator. Long-term follow-up. Rev Esp Cardiol. 2005:58(5):491-8.

12. El-Shalakany A, Hadjis T, Papageorgiou P, Monahan K, Epstein L, Josephson ME. Entrainment/mapping criteria for the prediction of termination of ventricular tachycardia by single radiofrequency lesion in patients with coronary artery disease. Circulation 1999;99(17): 2283-9.

13. Ávila $\mathrm{P}$, Arenal A. Substrate ablation of post-infarction ventricular tachycardias. Rev Española Cardiol. (english ed.) 2014;67(7):514-518.

14. Carbucicchio C, Santamaria M, Trevisi N, Maccabelli G, Giraldi F, Fas sini $\mathrm{G}$, et al. Catheter ablation for the treatment of electrical storm in patients with implantable cardioverter-defibrillators: short- and long-term outcomes in a prospective single-center study. Circulation 2008;117(4):462-9.

15. Atienza F, Arenal A, Pérez-David E, Elízaga J, Ortuño JE, Ledesma-Carbayo MJ, et al. New diagnostic and therapeutic approaches to trea ventricular tachycardias originating at the summit of the left ventricle: role of merged hemodynamic-mri and alternative ablation sources. Circ Arrhythm Electrophysiol. 2013;6(6):e80-4.

16. García-Morán E, Sandín-Fuentes MG, Álvarez López JC, Duro-Aguado I, Urueña-Martínez N, Hernández-Luis $\mathrm{C}$. Electrical storm secondary to acute myocardial infarction and heart failure treated with left stellate ganglion block. Rev Esp Cardiol (engl. ed) 2013;66(7):595-7.

17. Baratto F, Pappalardo F, Oloriz T, Bisceglia C, Vergara P, Silberbauer J, et al. Extracorporeal membrane oxygenation for hemodynamic support of ventricular tachycardia ablation. Circ Arrhythm Electrophysiol. 2016;9(12):e004492.
18. Tung $R$, Vaseghi M, Frankel DS, Vergara P, Di Biase L, Nagashima K et al. Freedom from recurrent ventricular tachycardia after catheter ablation is associated with improved survival in patients with structural heart disease: an International VT Ablation Center Collaborative Group study. Heart Rhythm 2015;12(9):1997-2007.

19. Izquierdo M, Ruiz-Granell R, Ferrero A, Martínez A, Sánchez-Gomez J, Bonanad $\mathrm{C}$, et al. Ablation or conservative management of electrical storm due to monomorphic ventricular tachycardia: differences in outcome. Europace 2012;14(12):1734-9.

20. Peichl P, Cihák R, Kozeluhová M, Wichterle D, Vancura V, Kautzner J. Catheter ablation of arrhythmic storm triggered by monomorphic ectopic beats in patients with coronary artery disease. J Interv Card Electrophysiol 2010;27(1):51-9.

21. Organización Nacional de Trasplante. 2017. Available at: http://www.ont. es/infesp/Paginas/Documentaciion.aspx.

22. Yokokawa M, Kim HM, Baser K, Stevenson W, Nagashima K, Della Bella $P$, et al. Predictive value of programmed ventricular stimulation after catheter ablation of post-infarction ventricular tachycardia. J Am Coll Cardiol. 2015;65(18):1954-1959.

23. Fernández-Armenta J, Penela D, Acosta J, Andreu D, Evertz R, Cabrera M, et al. Substrate modification or ventricular tachycardia induction, mapping, and ablation as the first step? A randomized study. Heart Rhythm 2016;13(8):1589-1595.

24. Ruiz Hernandez PM, Loughlin G, Arenal A. Elimination of all inducible ventricular tachycardias as the endpoint for ablation. J Am Coll Cardiol. 2015;66(22):2576

25. Di Biase L, Santangeli P, Burkhardt DJ, Bai R, Mohanty P, Carbucicchio C, et al. Endo-Epicardial homogenization of the scar versus limited substrate ablation for the treatment of electrical storms in patients with ischemic cardiomyopathy. J Am Coll Cardiol. 2012;60(2):132-141.

26. Nogami A. Mapping and ablating ventricular premature contractions that trigger ventricular fibrillation: trigger elimination and substrate modification. J Cardiovasc Electrophysiol. 2015;26(1):110-115.

27. Acosta J, Fernández-Armenta J, Penela D, Andreu D, Borras R, Vassanelli $F$, et al. Infarct transmurality as a criterion for first-line endo-epicardial substrate guided ventricular tachycardia ablation in ischemic cardiomyopathy. Heart Rhythm 2016;13(1):85-95.

28. García Carreño J, Sousa Casasnovas I, Vicent Alaminos ML, Atienza Fernández F, Martínez Sellés M, Fernández Avilés F. Utilizacion del oxigenador extracorporeo de membrana en pacientes con tormenta electrica: experiencia de un centro terciario. Rev Esp Cardiol. 2019;72(2):182-183. 